\title{
Hidden infrared star clusters in our Galaxy: follow-up observations
}

\section{Jura Borissova, ${ }^{1}$ Radostin Kurtev, ${ }^{1}$ Margaret M. Hanson, ${ }^{2}$ Leonid Georgiev, ${ }^{3}$ Valentin Ivanov $^{4}$ and Sergio Ortolani ${ }^{5}$}

${ }^{1}$ Departamento de Física y Astronomía, Facultad de Ciencias, University of Valparaíso, Chile

${ }^{2}$ Department of Physics, University of Cincinnati, Cincinnati, OH, USA

${ }^{3}$ UNAM, Mexico ${ }^{4}$ European Southern Observatory, Santiago 19001, Chile

${ }^{5}$ Dipartimento di Astronomia, University of Padova, Padova, Italy

\begin{abstract}
We are reporting some recent results from our long-term program aimed at characterizing the obscured present-day star cluster population in the Galaxy. Our goal is to expand the current census of the Milky Way's inner stellar disk to guide models seeking to understand the structure and recent star-formation history of our Galaxy. The immediate goal is to derive accurate cluster physical parameters using precise infrared photometry and spectroscopy. So far, we observed approximately 60 star cluster candidates selected from different infrared catalogs. Their nature, reddening, distance, age and mass are analyzed. Two of them, Mercer 3 and Mercer 5, are new obscured Milky Way globular clusters. Among the newly identified open clusters, the objects [DBS2003] 179, Mercer 23, Mercer 30, Mercer 70, and [DBS2003] 106 are particularly interesting because they contain massive young OB and Wolf-Rayet stars with strong emission lines.
\end{abstract}

Keywords. open clusters and associations: general, open clusters and associations: individual ([DBS2003]179, [DBS2003]106, [MCM2005b]23, [MCM2005b]30, [MCM2005b]70), globular clusters: individual ([MCM2005b]3, [MCM2005b]5), stars: early-type, stars: Wolf-Rayet

The full poster (in pdf format) is available at http://www. astro.iag.usp.br/ iaus266/Posters/pBorissova.pdf. 